\title{
Life skills training and booster sessions plus drinking reduction treatment was effective in heavily drinking women
}

\author{
Connors GJ, Walitzer KS. Reducing alcohol consumption among heavily drinking women: evaluating the contributions of \\ life-skills training and booster sessions. J Consult Clin Psychol 2001 Jun;69:447-56.

\begin{abstract}
QUESTION: In problem drinking women without histories of severe physical dependence on alcohol, does the addition of life skills training and booster sessions to drinking reduction treatment (DRT) reduce alcohol consumption?
\end{abstract}

\section{Design}

Randomised \{allocation concealed*\}†, blinded (outcome assessors)*, controlled trial with 18 months of follow up.

\section{Setting}

A university in Buffalo, New York, USA.

\section{Participants}

Source of funding: National Institute on Alcohol Abuse and Alcoholism

For correspondence: $D r$ G J Connors, Research Institute on

Addictions/University at Buffalo, The State University of New York, 1021 Main Street, Buffalo, NY 14203,

USA.

connors@ria.buffalo.edu.
144 self referred women who were $\geqslant 21$ years of age (mean age $39 \mathrm{y}$ ), drank $\geqslant 15$ drinks/week or had $\geqslant 2$ drinking days/week of 6 drinks/day, were interested in reducing their alcohol consumption, scored in the lower half of the Alcohol Dependence Scale (ie, moderate levels of alcohol dependence symptoms), had normal liver function tests, and were not pregnant. Exclusion criteria included previous hospital admission for drinking or drug use, previous detoxification from alcohol or any other drug, current alcohol related legal charges, current psychiatric treatment, and hospital admission in the past 5 years. $92 \%$ of women provided complete follow up drinking data.

\section{COMMENTARY}

The study by Connors and Walitzer shows that treatment reduces drinking in the subgroup of women who are mildly or moderately dependent heavy drinkers and that enhancing the treatment reduces it more. In addition, these changes in behaviour are well maintained through an 18 month follow up. The 2 enhanced treatments, however, are not comparable, because of their different timescales, with one lasting 10 weeks, and the other extending over 8 months. Time is an important ingredient in the change process, and indeed is shown to be an independent variable in this paper. Alternatively, it could be a simple "dose-response" relations with the"booster"groups having had more total effective treatment time.

It is also disappointing to see the high intensity of the input used. Those of us involved with treatment programmes in the real world will groan slightly to see that $>8$ hours of skilled therapist time was devoted to each participant in this study, even before enhancements, and even after taking account of the fact that the treatment was given in small groups. Given the population prevalence of this type of drinking at $4-7 \%$, this is an ambitious amount of time to devote to each person.

The presentation of the data in the article makes the results hard to understand in any detail. However, it seems that, at least in the heavier drinkers, all interventions were effective. Part of the purpose of the study was to test an intervention specifically targeted at women (life skills training). This did work, and was certainly better than general health education (the control condition) given for 7 hours during a 20 hour treatment reduction programme.

The study raises more questions than it answers. For example, how skilled do the therapists need to be? It would be important to know if the treatment could be done by district nurses in primary care. How intensive does the treatment need to be, and could "self-help" manuals be used to reduce direct therapist input? Could it be that the length of treatment (rather than the type) is the crucial variable? If so, would fewer sessions over a longer time period be more effective than the intensive 10 week treatment used here? Pete Sudbury, BM, BCh, MRCPsych, MBA Berkshire Healthcare NHS Trust Berkshire, UK

\section{Intervention}

Women were allocated to DRT alone $\{\mathrm{n}=31\} \uparrow$, DRT plus life skills training $\{n=39\}$, DRT plus booster sessions $\{\mathrm{n}=41\}$, or DRT plus life skills training and booster sessions $\{\mathrm{n}=33\} \uparrow$. Treatment was given in weekly 2 hour sessions in groups of 3-6 women over 10 weeks. Each group received 13 hours of DRT plus 7 hours of life skills training or a control treatment (alcohol and general health related lectures and videotapes). DRT involved teaching strategies for reducing and controlling alcohol intake and target exercises for homework. Life skills training included relaxation, problem solving, assertiveness skills, communication training, and target exercises for homework. Booster sessions were given in person or by phone at $2,4,7,10,13,16,20$, and 24 weeks after treatment.

\section{Main outcome measure}

Number of abstinent or light (AL) (1-3 standard drink equivalents [SDE]) drinking days. $1 \mathrm{SDE}$ was defined as $1 \mathrm{oz}(30 \mathrm{ml})$ of $86-100$ proof beverage or its equivalent in beer or wine (where $1 \mathrm{SDE}=1.5$ units of alcohol).

\section{Main results}

The initial analysis showed interactions among treatment groups and baseline drinking level. In women who were heavier drinkers at baseline (below the median number of AL drinking days $[18.4 \mathrm{~d} / \mathrm{mo}]$ ), those who received life skills training, booster sessions, or both reported more AL drinking days after treatment than those who received DRT alone $(\mathrm{p}<0.05)$. Women who received both life skills training and booster sessions in addition to DRT reported more AL drinking days than did women who received only 1 enhancement in addition to DRT throughout follow up $(\mathrm{p}<0.01)$; $\{$ the mean number of AL drinking days were 18.0 for DRT alone, 22.0 for DRT plus skills, 19.9 for DRT plus booster, and 26.8 for DRT plus both enhancements\}t. In women who were lighter drinkers at baseline ( $>18.4 \mathrm{AL}$ drinking days/mo), each group improved over time with no difference among treatment groups. Changes were maintained during the 18 months of follow up.

\section{Conclusions}

In problem drinking women without severe physical dependence on alcohol, the addition of life skills training and booster sessions enhanced the outcome of drinking reduction treatment by reducing alcohol consumption in those who drank heavily at baseline. In lighter drinkers, treatment groups did not differ.

*See glossary.

$\dagger$ Information provided by author. 